\title{
Information Needs for an Online Resource for LGBTQ+ Young People: Mental Health, Sexual Health, and Navigating Services
}

Daniel Delmonaco is a PhD student in the School of Information at the University of Michigan.

Shannon $\mathrm{Li}$ is an undergraduate student of information at the University of Michigan.

Christian Paneda is a graduate student of humancomputer interaction at the University of Michigan.

Luna Hughson is the CHAl Lab Project Coordinator at the University of Michigan.

Elliot Popoff is a Project Director at the University of Michigan School of Public Health.

Laura Jadwin-Cakmak is a Research Director at University of Michigan School of Public Health.

Gary W. Harper, MPH is a Professor in the Department of Health Behavior and Health Education, as well as a Professor of Global Public Health.

Dr. Oliver Haimson ${ }^{\dagger}$ is an assistant professor of information at the University of Michigan. 
Delmonaco, D., , Li, S., Paneda, C., Hughson, L., Popoff, E., Jadwin-Cakmak, L., Harper, G.W. \& Haimson, O. (2021). Information Needs for an online resource for LGBTQ+ young people: Mental health, sexual health, and navigating services. Currents, (2)1.

Keywords: mental health, lgbtq + mental health, child mental health, LGBTQ+ Health

Gender and sexual-minority youth have unique health needs that are often underserved by institutions such as school sex education curricula and health-care providers (Charest et al., 2016; Haley et al., 2019; Mitchell et al., 2014). In this paper, we describe our ongoing work to address these disparities with the collaborative development of health resources, emphasizing mental and sexual health for lesbian, gay, bisexual, transgender, and queer and/or questioning (LGBTQ+) youth. Our community partner is the Community Health Access Initiative (CHAl) at the University of Michigan. The goal of CHAl is to improve and support LGBTQ+ young people's health through improving access to affirming health care. With this collaboration between $\mathrm{CHAl}$ and social-computing researchers, we are in the process of developing an online resource for LGBTQ+ youth providing comprehensive healtheducation information. In this paper, we describe part of our research and design process and also present topics that participants identified as vital to include in a resource such as this intended website. The idea for a website resulted from CHAl's Action Committee, a group of LGBTQ+ young-adult advisors to the project, who identified a serious need for relevant and inclusive health information that they and their peers struggled to find online and offline. While the current CHAI website includes substantial resources and references to services, CHAl is looking for further ways the site can meet LGBTQ+ young people's needs. For instance, the site could include more comprehensive resources, ranging from mental health to physical services. 
Additionally, the site aims to improve how resources are organized to optimize usability, navigability, and accessibility.

LGBTQ+ people face substantial health disparities compared to the general population (James et al., 2016; Trevor Project, 2020; Juster, 2017). In a 2020 national survey of LGBTQ+ youth in the United States, the Trevor Project reported that $40 \%$ of LGBTO respondents ages 13 to 24 seriously considered attempting suicide, and $68 \%$ reported symptoms of generalized anxiety disorder (Trevor Project, 2020). Transgender and nonbinary youth reported even higher instances of these mental health indicators (Trevor Project, 2020). Additionally, young LGBTQ+ people face many difficulties accessing relevant health resources to address these needs due to homophobia, transphobia, privacy concerns, and fear for safety if outed as a gender or sexual minority (Patterson et al., 2019). We seek to understand how LGBTQ+ youth use online resources to meet their healtheducation information needs, particularly related to mental health and sexual health. In this paper, based on analysis of three focus groups with LGBTQ+ youth, we describe three topics participants described as vital for an online resource: mental health resources, sexual health resources, and resources for navigating services. Understanding the online health-information practices of LGBTQ+ youth will directly inform the development of a new website connecting LGBTQ+ youth with relevant resources on topics identified by our participants.

\section{Methods}

We conducted three virtual focus groups with 11 self-identified LGBTQ+ young people ages 16 to 24 . This age range is consistent with CHAl's Action Committee of young community members and the young people they provide services to. Participants were recruited using recruitment emails and convenience sampling in coordination with our community partner. Each focus group included three to four participants, for a total of 11 participants. Focus groups began 
with semistructured group interviews to discuss experiences seeking health information and desired information they would like to see on our forthcoming website. We followed the interview component with design sessions in which participants used digital drawing tools or their own materials (e.g., pen and paper) to sketch designs for their ideal LGBTQ+ health web resource. This study received approval from the University of Michigan Institutional Review Board.

All focus groups were recorded and transcribed. Two authors used an inductive open-coding approach (Strauss \& Corbin, 1998) to code focus group transcripts and identify themes in the data. Main themes we identified included specific content participants identified as necessary for CHAl's website, difficulties accessing health information, and experiences seeking relevant health information online via social media and search engines. We also used sketches and designs from focus groups to develop website prototypes. Figures 1 and 2 are examples of designs created in focus groups that informed our development process.

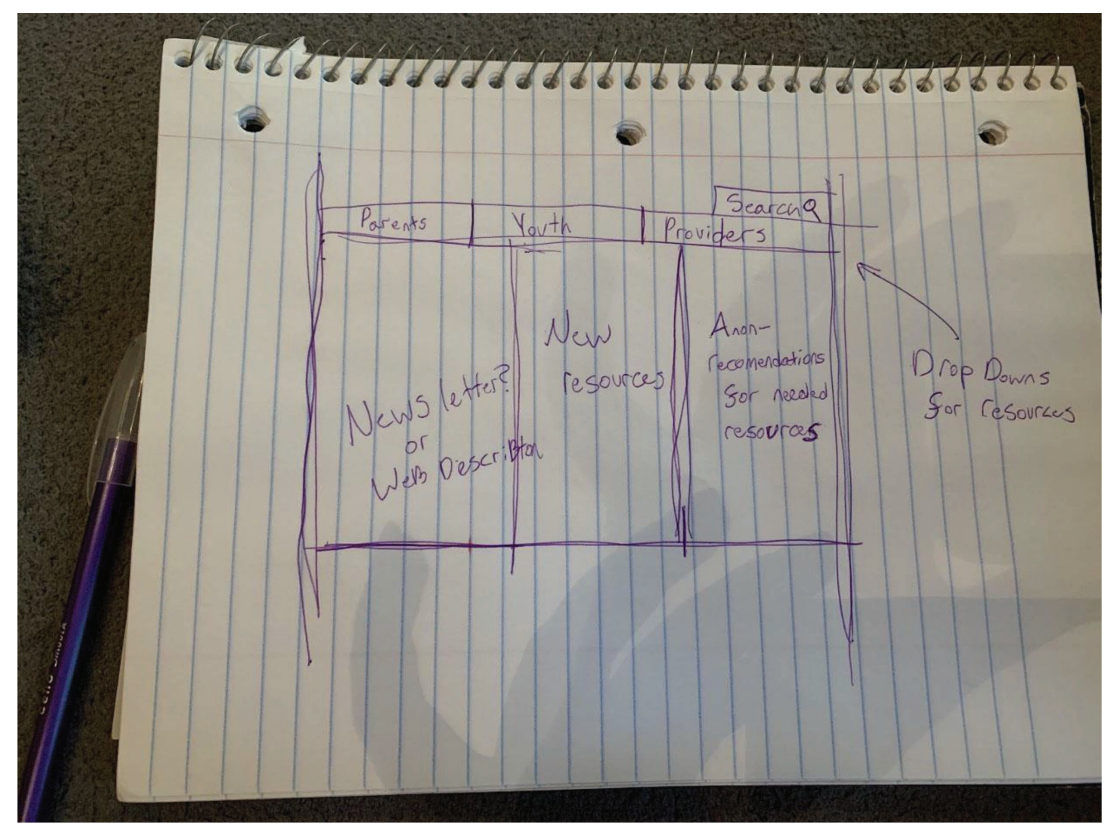

Figure 1. Courtesy of Dan Delmonaco 


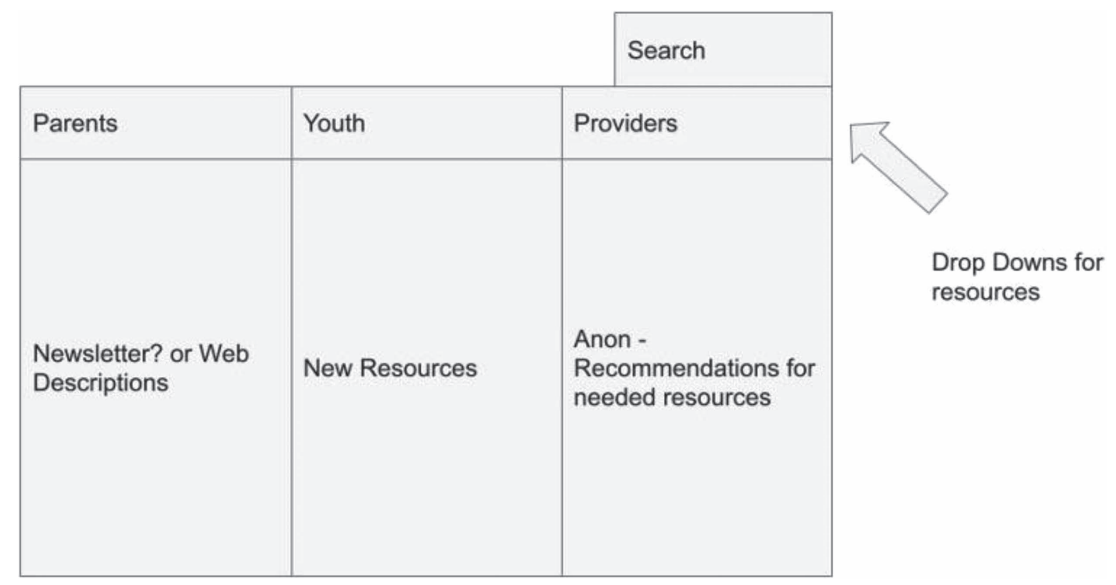

Figure 2. Courtesy of Dan Delmonaco

Our research team met with $\mathrm{CHAl}$ to collaboratively analyze data, discuss emerging results, and present designs throughout the dataanalysis process. Once themes were identified, we used the card sorting technique ${ }^{1}$ to organize relevant health topics mentioned in focus groups to determine how they should be organized on the new website. Focus group participants completed card sorting tasks using Optimal Workshop, an online card sorting tool. In our "Results" section, we present key areas participants identified as necessary for inclusion on the CHAl website.

The third author designed the website using a human-centered design process. The website and mobile application equivalent are currently in development (see Figure 3 and Figure 4). Our iterative design process includes regular team meetings with $\mathrm{CHAl}$, frequent feedback, and user testing with focus group participants.

1. Card sorting is a method that enables researchers to understand how people categorize concepts. 


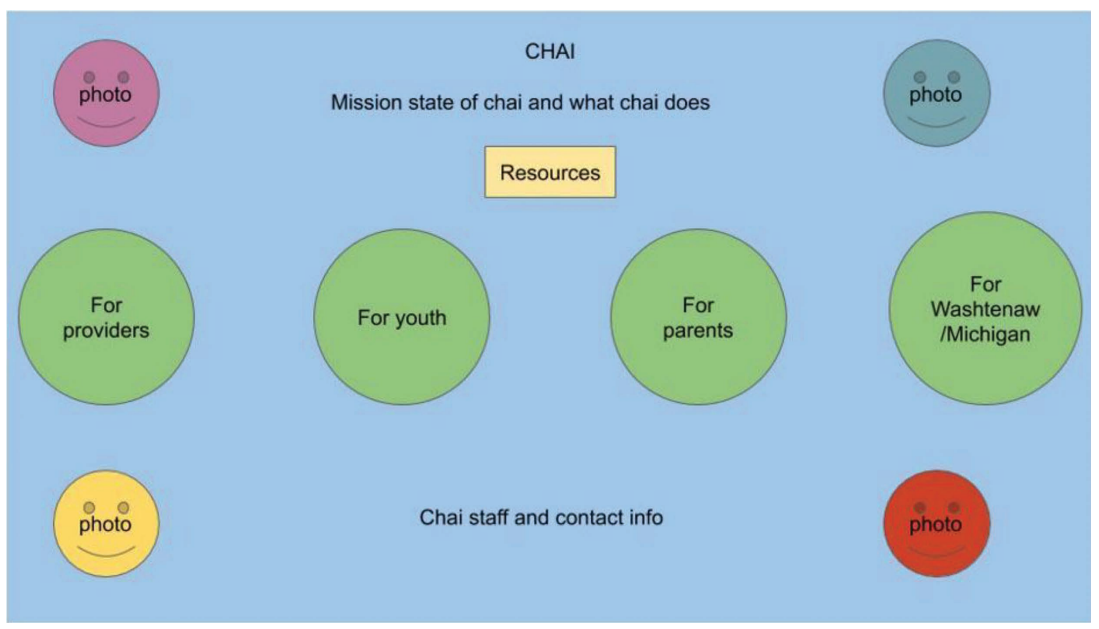

Figure 3. Courtesy of Dan Delmonoco

\section{Results}

Due to the difficulties so many young LGBTQ+ people face when seeking relevant health information, participants identified many health-care topics to include on the CHAl website for LGBTQ+ youth. In this section, we present three health topic areas discussed by participants in all virtual focus groups: mental health resources, sexual health resources, and resources for navigating services.

\section{Mental Health Resources}

Participants described their experiences seeking mental health services and the difficulties faced, especially as LGBTQ+ minors. For those living with family or guardians as minors or due to other circumstances, it was often especially difficult to access mental health services when in an unsupportive and potentially dangerous environment. P3 expressed some of these concerns: 

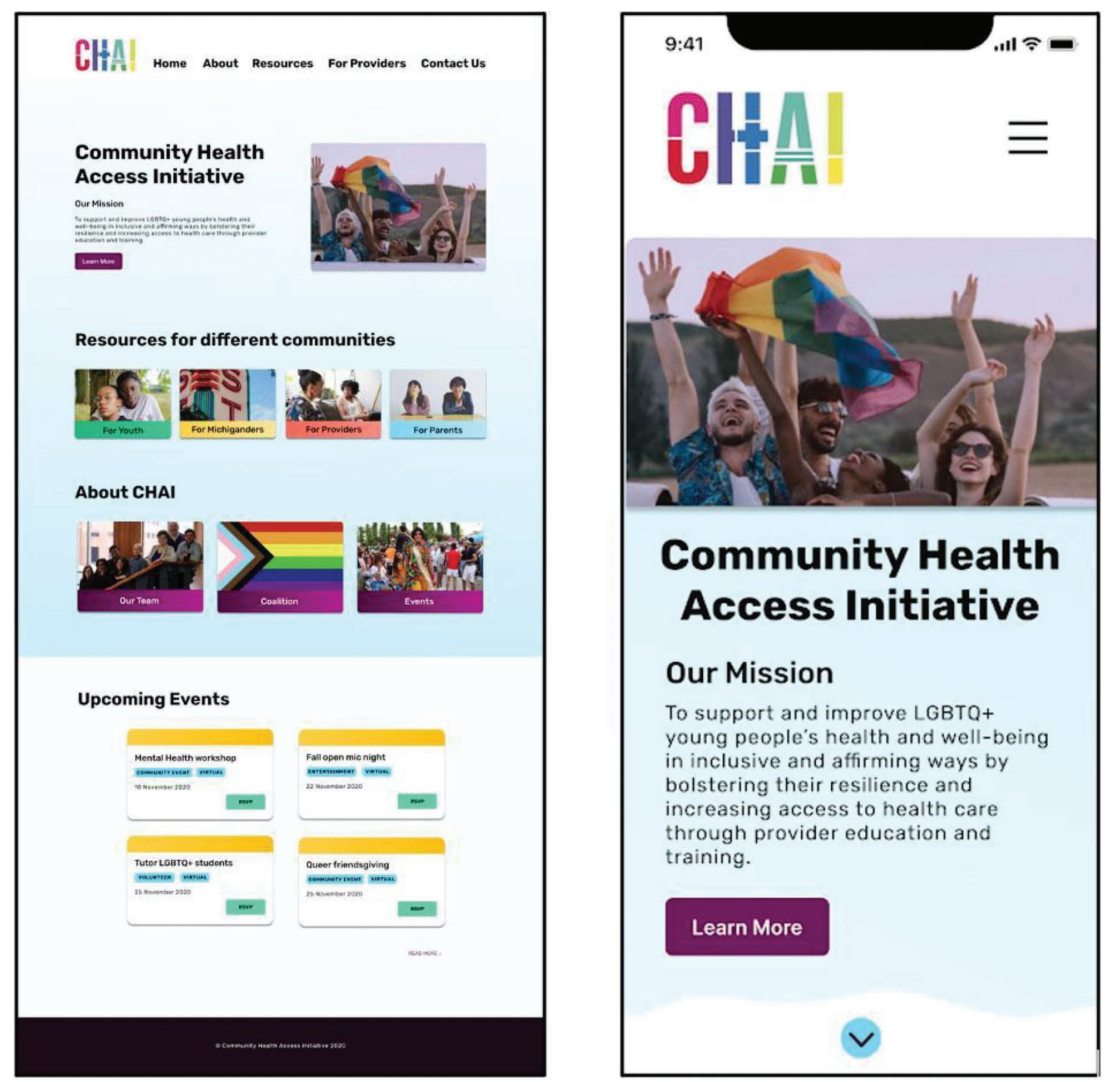

Figure 4. Courtesy of Dan Delmonaco

I don't know what options exist for if you want to take more control over your mental health, but you can't take yourself out of a toxic situation. So I'm curious about what kind of resources would be available there. Especially since we mentioned living in an environment that might not be supportive of us, being our whole selves. (P3, 23, Nonbinary, Pansexual)

P3's concerns are some we hope to address in the development of the $\mathrm{CHAl}$ website. In addition to providing information about accessing mental health services, it will be important to also address improving 
and supporting LGBTQ+ young people's mental health while in situations often outside of their own control. For instance, LGBTQ+ youth may find support via an LGBTQ+ community. P1 mentioned the importance of community to LGBTQ+ young people:

I think having some way to provide kids with resources to those local groups or communities is super important. I know that was in one of my first therapy appointments, we talked about my goals and one of them was wanting to be more a part of the LGBT community in [current US city] because I felt so disconnected and just lonely. And that was a huge part of my mental health struggle. So I think having a community is a huge thing and it's a massive part of health. So I think giving those resources to find those communities locally would be really important. (P1, 22, Female, Lesbian)

P1 found a mental health professional to work with but knew that finding an "LGBT" community would be an important part of improving their overall mental health. The website we develop needs to incorporate the complexities of mental health care and take a holistic approach to providing resources. Finding a mental health professional is one component, but other important strategies include identifying potential online and offline communities for support and connecting people with these resources.

\section{Sexual Health Resources}

Sex education curricula and health-care providers did not meet participants' sexual health information needs. Participants pointed to online resources as vital for sex and sexual health information seeking. They described many sexual health topics to cover on the website, including trans-specific sexual health information, sexually transmitted infection (STI) testing, and navigating online dating.

Identity and labels related to sexuality informed some recommendations made by participants. Participants described the importance 
of understanding terminology related to gender, sexuality, and sexual health. P9 shared:

For example, if you're defining the term bisexual, I would say make sure to make it known that you don't have to identify the same way as another person who's bisexual to call yourself bisexual. And you can define your own label. ... Of course, it's very common, it's completely okay to go through a bunch of labels or not have a label all depending on what you're most comfortable with. But I found it where people were shaming of you if you weren't identifying the exact same way that they thought that a bisexual, for example, should label themselves. (P9, 22, Nonbinary, Pansexual)

In the case of P9, there was a desire for a more inclusive understanding of the word bisexual to be presented to other young people. It is important to note in P9's response and those shared by others that providing definitions should come with the acknowledgment that identities related to gender and sexuality are fluid and labels may change.

Participants were particularly enthusiastic about consent resources. P4 explained the importance of consent and why it should be included on our website:

I also think something that would have been really important would have been resources for how to navigate sexual and medical boundaries. Straight up specific exercises, like practice this with a friend or with a dog or with whatever. Practice saying yes, practice saying no, practice asking for consent, practice being told yes or no in regards to that. And really normalizing that is healthy and respectful to yourself and to the person that you're with. (P4, 21, Female, Bisexual or Pansexual)

To P4, consent was an important concept for navigating relationships and their own boundaries that they needed to figure out on their own so they can establish safe relationships, which is crucial to maintaining healthy emotional well-being. Including this topic on the website might help introduce the idea of consent and autonomy of one's own 
body to LGBTQ+ young people, particularly since this information is usually not available elsewhere in their lives.

\section{Resources for Navigating Services}

Difficulties accessing resources were present throughout the discussions of mental health and sexual health. Participants described hoping that the CHAl website would provide clear information about accessing health care as a young LGBTQ+ person.

A major aspect of accessing medical services is navigating insurance, as P7 mentioned: "One thing I feel like would definitely be very helpful is how to navigate different social services. Like applying for Medicaid." (P7, 23, Female, Bisexual). P7 specifically referenced Medicaid as one component of insurance and LGBTQ+ health care that could be explained on our website. Participants also discussed wanting resources for figuring out insurance and costs as a minor, especially if trying to access care without contacting one's parent/guardian. For example, a young person might want to have a sexually transmitted infections (STI) test but cannot afford it on their own and cannot let their parents know due to unwanted disclosure and/or fear of punishment. On our website, we can explain possibilities for accessing STI testing without using parents' insurance, such as via a local LGBTQ+ nonprofit.

Another issue participants described wanting to see addressed is difficulty finding an LGBTO+ inclusive health-care provider. P2 explained difficulties they faced when moving to a new place:

This past year I moved from [one region of the United States to another]. So I had to find all new doctors and find doctors that worked with my insurance. I tried to find a general practitioner who was maybe knowledgeable about other sexualities or identities and I moved to conservative [US region]. So that's not really something that worked out for me. And it was kind of just a crap shoot to choose a doctor. I do like the doctor that I ended up with, but there wasn't really any sort of resource for me to go to, to find someone or at least not a resource 
I was able to find that would help me find a doctor who specifically catered to that side of me even. It's not even in a health way, but just someone you can talk to about either sex ed stuff or gender stuff. (P2, 22, Questioning/AFaB [assigned female at birth], Biromantic asexual)

P2 luckily found a doctor but did not have much control over this experience, largely due to lack of resources. Participants discussed struggles finding health-care providers to tend to their specific health needs as LGBTQ+ people, as most providers are not educated to adequately treat LGBTQ+ patients (Keuroghlian, 2017). Some ideas mentioned were a verified directory for LGBTQ+ inclusive providers on the website and interactive maps with provider information and options for rating and recommending providers within the interface.

As we transition from the ideation phase of the focus groups to the development and eventual deployment of the CHAl website, we hope to address these three areas of concern and other topics expressed by participants in our study.

\section{Implications}

The topics we identified in the focus groups and participants' website designs directly inform our development of the CHAl website and mobile application and the resources available to LGBTQ+ young people. Mental health, sexual health, and resources for navigating services were major areas in which participants identified disparities and difficulty accessing relevant resources. Identifying LGBTQ+ young people's needs for an online resource has been instrumental in our ongoing work designing and developing the new $\mathrm{CHAl}$ website and mobile application, which will fill an informational gap and help to address disparities faced by LGBTQ+ young people. In developing this resource, we aim to equip LGBTQ+ young people with tools accessible for a variety of electronic devices to take care of themselves and advocate for their health needs within health-care settings in which access to services is difficult to reach. 


\section{Biographies}

Daniel Delmonaco's research focuses on the ways that LGBTQIA+ young people use online resources to identify and meet information needs related to sex and sexual health.

Gary W. Harper's research and community work focuses on the mental health and sexual health needs of adolescents and young adults who experience varying degrees of oppression and marginalization.

Shannon Li is an undergraduate student of information at the University of Michigan.

Christian Paneda is a graduate student of human-computer interaction at the University of Michigan.

Luna Hughson is the CHAl Lab Project Coordinator at the University of Michigan.

Elliot Popoff is a Project Director at the University of Michigan School of Public Health.

Laura Jadwin-Cakmak is a Research Director at University of Michigan School of Public Health.

Dr. Oliver Haimson's research focuses on envisioning and designing trans technologies, social media content moderation, and marginalized populations.

\section{References}

Charest, M., Kleinplatz, P. J., \& Lund, J. I. (2016). Sexual health information disparities between heterosexual and LGBTQ+ young adults: Implications for sexual health. Canadian Journal of Human Sexuality, 25(2), 74-85. 
Haley, S. G., Tordoff, D. M., Kantor, A. Z., Crouch, J. M., \& Ahrens, K. R. (2019). Sex education for transgender and non-binary youth: Previous experiences and recommended content. The Journal of Sexual Medicine, 16(11), 1834-1848. https://doi.org/10.1016/j.jsxm.2019.08.009

James, S. E., Herman, J. L., Rankin, S., Keisling, M., \& Mottet, L, \& Ma'ayan, A. 2016. The report of the 2015 U.S. transgender survey. National Center for Transgender Equality, Washington, DC.

Juster, R. P., Vencill, J. A., Johnson, P. J. (2017) Impact of stress and strain on current LGBT health disparities. In Eckstrand K., Potter J. (Eds.). Trauma, Resilience, and Health Promotion in LGBT Patients. Springer. https://doi. org/10.1007/978-3-319-54509-7_4

Keuroghlian, A. S., Ard, K. L., \& Makadon, H. J. (2017). Advancing health equity for lesbian, gay, bisexual and transgender (LGBT) people through sexual health education and LGBT-affirming health care environments. Sexual Health, 14(1), 119-122. https://doi.org/10.1071/SH16145

Mitchell, K. J., Ybarra, M. L., Korchmaros, J. D., \& Kosciw, J. G. (2014). Accessing sexual health information online: Use, motivations and consequences for youth with different sexual orientations. Health Education Research, 29(1), 147-157. https://doi.org/10.1093/her/cyt071

Patterson, S. P., Hilton, S., Flowers, P., \& McDaid, L. M. (2019). What are the barriers and challenges faced by adolescents when searching for sexual health information on the internet? Implications for policy and practice from a qualitative study. Sexually Transmitted Infections, 95(6), 462-467. https://doi.org/10.1136/sextrans-2018-053710

Strauss, A., \& Corbin, J. M. (1998). Basics of Qualitative Research: Techniques and Procedures for Developing Grounded Theory. SAGE Publications.

Trevor Project. (2020). 2020 national survey on LGBTQ youth mental health. Trevor Project. 